\title{
Fixed-Time Synchronization of Drive-Response Coupled Systems with Impulsive Effects
}

\author{
Wenjing Yang \\ College of Science, University of Shanghai for Science and Technology, Shanghai, P. R. China \\ Email: wenjingyangmail@163.com
}

\begin{abstract}
In this paper, the fixed-time synchronization for drive-response coupled system(DRCS) with impulsive effects is investigated. More general controllers are designed to synchronize driveresponse coupled system(DRCS) within fixed-time. By using graph theory and Lyapunov method, strongly and non-strongly connected of topological structure of DRCS are studied deriving different criteria. What's more, some control strategies are provided for special cases of DRCS. Furthermore, some numerical simulations are offered to demonstrate the validity of theoretical results.
\end{abstract}

Keywords: Fixed-time synchronization, Drive-response coupled system, Impulsive effects, Graph theory.

\section{Introduction}

During the past decade, coupled systems have received increasing attracted attention. The main reason is that many real system can be described as coupled systems in many fields including mathematic $[1,2]$, biology $[3,4,5]$, physics $[6,7]$. Actually, coupled systems also can be found in engineering including pattern recognition, image processing, big data and so on $[8,9,10]$.

Synchronization, an important dynamical behavior, indicates that two or more systems, which are either equivalent or nonequivalent systems, tend to a common state as the network evolves by a suitable coupling or forcing [11]. What's more, many kinds of synchronization have been extensively studied due to its various applications, such as complete synchronization [12], projective synchronization [13], exponential synchronization [14], asymptotic synchronization [15]. Recently, synchronization of coupled systems has been a subject of active research due to the fact that many natural phenomena have close relationships with system synchronization [16]. For its potential applications in various fields, some intriguing results have been reported $[17,18,19,20]$. Pecora and Carroll were the first to study the synchronization of drive-response systems in [21]. This method requires that the drive system can break down a subsystem and then copy it, and the resulting system is called a response system. Studying classic chaos systems often uses this method, such as Lorenz system, chua circuit system. In communication security and neuroscience, the synchronization of the drive-response systems has been extensively investigated in view of its wide applications. An example comes from the computer world. All educators' computers form educational networks, while networks of all researchers form research networks. The two networks are coupled over the Internet. If all educators and researchers explore the same source of the Internet, congestion will occur, which is harmful and should be avoided. Therefore, it is necessary to study the synchronization between coupled networks. Therefore, [20] study synchronization between two coupled complex network with same topologies.

The convergence rate of the synchronization problem, which means that synchronization can be achieved as quickly as possible, is an important topic in numerous practical applications. Many researchers investigated asymptotical and exponential synchronization of network [15,22]. Whereas most of the existing important results on asymptotic and exponential synchronization were defined over an infinite time interval. Only when time approaches to infinity, the drive-response system can realize synchronization. Since the life span of biologies and machine are limited, minimizing the synchronization time is of essence in achieving fast communication synchrony. In order to enhance convergence, finite-time synchronization proposed in [23] had optimal convergence time. Moreover, finite-time control exhibits better disturbance rejection properties compared with asymptotic control [24]. Hence, finite-time synchronization has been widely studied in recent years $[25,26,27,28]$. 
It should be noted that the convergence time of finite-time control approach is heavily dependent on the initial conditions of considered systems, which means the convergence time not fixed for different initial values. Moreover, it is too hard to adjust or even impossible to estimate the initial conditions of many practical systems. This deteriorate the systems' performance. These drawbacks prohibit the practical application of finite-time techniques. To overcome this setback, the definition of fixed-time control was firstly introduced in [29], which proposed nonlinear feedback design for the fixed-time stabilization of linear control systems. The advantage of fixed-time techniques over finite-time ones is that the convergence time is independent on the initial states of considered systems, Therefore, the parameters of designed controllers completely determine the convergence time of fixed-time techniques. In view of the framework of fixed-time techniques, many new control schemes are established in $[30,31,32,33]$. Especially, fixed-time derive-response synchronization of Cohen-Grossberg neural networks was considered in [32].

In the process of signal transmission, networks may experience abrupt changes in their structure and parameters caused by component failures or repairs. This is, the controlled network may exhibit impulsive effects [34,35]. Impulsive system is comprised of three necessary parts, i.e., a continuous differential equation, which governs the evolution of the system between impulsive events; an impulse state jumping function, which describes the way the states of the system are instantaneously jumped; and a criterion which determines when the states of the system are to be changed. A good deal of literature has been available to discuss the theory for impulsive different systems, see $[36,37,38]$. In [36], the authors studied the periodic solutions problem for impulsive differential equations. The fixed-time stabilization for impulsive Cohen-Grossberg BAM neural network in paper [37]. Hence, it is of great significance to take impulsive effects into consideration when studying the synchronization problem for DRCS. To the best of our knowledge, fixed-time synchronization of DRCS with impulsive effects has not been investigated in the exiting literature.

Motivated by the above discussions, synchronizing the drive-response coupled network with impulsive effects within fixed-time will be studied in this paper. The main contributions are as follows: (1) It is the first time that the fixed-time synchronization for the drive-response coupled network with impulsive effects is considered and some effective fixed-time synchronization criteria are derived; (2) Less conservative controllers are designed to cope with the difficulties induced by impulsive perturbations; (3) This paper considers two cases of topological structure of system, which are strongly connected and non-strongly connected graphs.

The remainder of this paper is outlined as follows. In section 2, some preliminaries and the model description are presented. In Section 3, the control strategies are considered for strongly connected and non-strongly connected topological structure of the drive-response coupled system with impulsive effects, respectively. Some simulation examples are given to confirm the efficiency of the proposed method in Section 4. Finally, Section 5 provides some conclusions.

Notation Let $R=(-\infty,+\infty)$ and $R^{+}=[0,+\infty), R^{n \times n}$ denotes the set of $n \times n$ real matrix. $\mathbb{N}^{+}$ denotes the sets of positive integers. For the vector $v \in R^{n}, v^{T}$ is defined as its transpose, $\|v\|$ is Euclidean norm and $\|v\|_{r}=\left(\sum_{i=1}^{n}\left|v_{i}\right|^{r}\right)^{\frac{1}{r}}$. For the matrix $A \in R^{n \times n}, A^{s}=\left(A+A^{T}\right) / 2,\|A\|=\sqrt{\lambda_{\max }\left(A^{T} A\right)}$, where $\lambda_{\max }\left(A^{T} A\right)$ is the maximum eigenvalue of $A^{T} A$. $\operatorname{diag}[\alpha]_{n}:=\operatorname{diag}\left(\alpha_{1}, \alpha_{2}, \ldots, \alpha_{n}\right) \in R^{n \times n}$ is the diagonal matrix with diagonal entries $\alpha_{i}, i=1,2, \ldots, n . I_{n} \in R^{n \times n}$ is the identity matrix. $\operatorname{sign}(z)(z \in R)$ is the sign function. Let $f(x)=\left(f_{1}\left(x_{1}\right), f_{2}\left(x_{2}\right), \cdots, f_{n}\left(x_{n}\right)\right)^{T}$, where $f_{i}: R \rightarrow R, x=\left(x_{1}, x_{2}, \cdots, x_{n}\right)^{T} \in R^{n}$. The sign matrix function $f$ is defined as

$$
\begin{aligned}
\operatorname{Sign}[f(x)-f(y)] & :=\operatorname{diag}\left[\operatorname{sign}\left(f_{i}\left(x_{i}\right)-f_{i}\left(y_{i}\right)\right]_{n},\right. \\
|f(x)-f(y)|^{\theta} & :=\left(\left|f_{1}\left(x_{1}\right)-f_{1}\left(y_{1}\right)\right|^{\theta}, \ldots,\left|f_{n}\left(x_{n}\right)-f_{n}\left(y_{n}\right)\right|^{\theta}\right)^{T}, \\
\operatorname{sign}(f(x)-f(y))^{\theta} & :=\operatorname{Sign}[f(x)-f(y)] \cdot|f(x)-f(y)|^{\theta} .
\end{aligned}
$$

\section{Model Formulation and Preliminaries}

In this paper, $\mathcal{G}=(\mathbb{L}, \mathcal{E})$ is introduced as a directed graph which contains a set $\mathbb{L}=\{1,2, \ldots, N\}$ of vertices and a set $\mathcal{E}$ of $\operatorname{arcs}(i, j)$ leading from vertex $j$ to $i . B=\left(b_{i j}\right)_{N \times N}$ is the weight matrix, where $b_{i j}>0$ if and only if $(i, j) \in \mathcal{E}$. The weight matrix $B$ is irreducible if and only if a weighted digraph 
$(\mathcal{G}, B)$ is strongly connected. The Laplacian matrix of $(\mathcal{G}, B)$ is defined as

$$
L=\left[\begin{array}{cccc}
\sum_{j \neq 1} b_{1 j} & -b_{12} & \cdots & -b_{1 N} \\
-b_{21} & \sum_{j \neq 2} b_{2 j} & \cdots & -b_{2 N} \\
\vdots & \vdots & \ddots & \vdots \\
-b_{N 1} & -b_{N 2} & \cdots & \sum_{j \neq N} b_{N j}
\end{array}\right]
$$

and $c_{i}$ is the cofactor of the $i$ th diagonal element of $L$. In particular, if $(\mathcal{Q}, B)$ is strongly connected, the $c_{i}>0$ for $i \in \mathbb{L}$.

Lemma 1. [1] Assume $N \geq 2$, then the following identity holds:

$$
\sum_{i, j=1}^{N} c_{i} b_{i j} F_{i j}\left(x_{i}, x_{j}\right)=\sum_{\mathcal{Q} \in \mathbb{Q}} W(\mathcal{Q}) \sum_{(s, r) \in \mathcal{E}\left(\mathcal{C}_{\mathcal{Q}}\right)} F_{i j}\left(x_{i}, x_{j}\right),
$$

where $F_{i j}\left(x_{i}, x_{j}\right): R^{m_{i}} \times R^{m_{j}} \rightarrow R$ are arbitrary functions, for any $i, j \in \mathbb{L}, \mathbb{Q}$ is the set of spanning unicyclic graphs of $(\mathcal{G}, B), W(\mathcal{Q})$ is the weight of $\mathcal{Q}$, and $\mathcal{C}_{\mathcal{Q}}$ stands for the directed cycle of $\mathcal{Q}$.

The following drive system, which is considered in this paper, define on a digraph $\mathcal{G}$ with $N(N \geq 2)$ vertices

$$
\dot{x}_{i}(t)=f_{i}\left(x_{i}(t)\right)+\rho \sum_{j=1}^{N} b_{i j} H_{i j}\left(x_{i}(t), x_{j}(t)\right)+u_{i}(t), i \in \mathbb{L},
$$

where $x_{i}(t)=\left(x_{i 1}(t), x_{i 2}(t), \ldots, x_{i m_{i}}(t)\right)^{T} \in R^{m_{i}}$ is the state variables of the $i$ th vertex system, $f_{i} \in R^{m_{i}}$ is the continuous function, $\rho>0$ is the coupling strength, $B=\left(b_{i j}\right)_{N \times N}$ is the weight configuration matrix, which stands for coupling strength. $H_{i j}: R^{m_{i}} \times R^{m_{j}} \rightarrow R^{m_{i}}$ is the continuous function describing coupling form. $u_{i}(t) \in R^{m_{i}}$ is the controller designed for the system (1). The initial value of the network (1) is $x_{i}(0)=x_{i 0}$.

We refer to model (1) as the drive coupled system and consider the following response system:

$$
\dot{y}_{i}(t)=f_{i}\left(y_{i}(t)\right)+\rho \sum_{j=1}^{N} b_{i j} H_{i j}\left(y_{i}(t), y_{j}(t)\right),
$$

where $y_{i}(t)=\left(y_{i 1}(t), y_{i 2}(t), \ldots, y_{i m_{i}}(t)\right)^{T} \in R^{m_{i}}$ is the response state of the $i$ th node with initial value $y_{i}(0)=y_{i 0}$.

The aim of this paper is to synchronize the drive-response systems (1) and (2). More precisely, the synchronization goal is described as following.

Definition 1. [30] The drive network (1) is said to be synchronized onto the response network (2) in a fixed time, if there exists a settling time $T>0$, which is independent of the initial value $x_{i 0}$ and $y_{i 0}$, such that

$$
\lim _{t \rightarrow T}\left\|x_{i}(t)-y_{i}(t)\right\|=0
$$

Then we define the synchronization error $e_{i}(t)=x_{i}(t)-y_{i}(t), i \in \mathbb{L}$. In the process of signal transmission, the states, $e_{i}(t), i=\mathbb{L}$, may be suddenly changed at some discrete time instants which can be described as a differential equation with impulses [26][39]. Hence, we have the following model with impulsive effects:

$$
\left\{\begin{array}{l}
\dot{x}_{i}(t)=f_{i}\left(x_{i}(t)\right)+\rho \sum_{j=1}^{N} b_{i j} H_{i j}\left(x_{i}, x_{j}\right)+u_{i}(t), t \neq t_{k} \\
\triangle x_{i}\left(t_{k}\right)=-\lambda_{i}^{k} e_{i}\left(t_{k}^{-}\right), t=t_{k}
\end{array}\right.
$$


where $\lambda_{i}^{k}$ are constants which denote the impulsive strength; the time sequence $\left\{t_{k}, k \in \mathbb{N}^{+}\right\}$, which satisfies $0<t_{1}<t_{2}<\ldots<t_{k} \ldots, \lim _{k \rightarrow \infty} t_{k}=\infty$, denotes the impulsive instants; $\triangle x_{i}\left(t_{k}\right)=x_{i}\left(t_{k}^{+}\right)-$ $x_{i}\left(t_{k}^{-}\right), x_{i}\left(t_{k}^{+}\right)$and $x_{i}\left(t_{k}^{-}\right)$denote the limit from the left and the right at time $t_{k}$, respectively. Without loss of generality, throughout this paper, we assume that $x_{i}\left(t_{k}^{+}\right)=\lim _{t \rightarrow t_{k}+0} x_{i}(t)=x_{i}\left(t_{k}\right)$, and $t_{0}=0$. Note that the impulsive effects $\lambda_{i}^{k}$ not only depend on the node $i$, but also rely on the impulsive instant $t_{k}$, which means that the impulsive effect can be distinct in both time domain and space domain [38].

The error dynamical system, which follows from the continuity of $y_{i}(t)$, is governed as follows:

$$
\left\{\begin{aligned}
\dot{e}_{i}(t)= & f_{i}\left(x_{i}(t)\right)-f_{i}\left(y_{i}(t)\right)+u_{i}(t) \\
& +\rho \sum_{j=1}^{N} b_{i j}\left(H_{i j}\left(x_{i}, x_{j}\right)-H_{i j}\left(y_{i}, y_{j}\right)\right), t \neq t_{k} \\
\triangle e_{i}\left(t_{k}\right) & =-\lambda_{i}^{k} e_{i}\left(t_{k}^{-}\right), t=t_{k}
\end{aligned}\right.
$$

where $\triangle e_{i}\left(t_{k}\right)=e_{i}\left(t_{k}\right)-e_{i}\left(t_{k}^{-}\right)$.

According to Definition 1, synchronizing the drive-response systems (3) and (2) within fixed-time is equivalent to stabilizing the origin of error system (4) in a fixed time.

In order to obtain the main results, The following definition, lemmas and assumptions are needed.

Definition 2. [40] Function $V: R^{n} \rightarrow R^{+}$is said to belong to class $\mathcal{V}$ if

(i) $V$ is continuous on each of the sets $\left[t_{k}, t_{k+1}\right) \times R^{n}$ for $x \in R^{n}, k \in \mathbb{N}^{+}$, and $\lim _{(t, u) \rightarrow\left(t_{k}^{-}, v\right)} V(t, u)=$ $V\left(t_{k}^{-}, v\right)$ exists;

(ii) $V$ is locally Lipschitzian in $x$.

Lemma 2. [41] If a positive definite, radially unbounded function $V(e(t)) \in \mathcal{V}$ such that any solution $e(t)$ of (4) satisfies

$$
\begin{aligned}
& \dot{V}(e(t)) \leq-\beta V^{q}(e(t))-\gamma V^{p}(e(t)), t \neq t_{k}, t \in R^{+}, \\
& V\left(e\left(t_{k}\right)\right) \leq V\left(e\left(t_{k}^{-}\right)\right), k \in \mathbb{N}
\end{aligned}
$$

where $\beta>0, \gamma>0,1>p>0, q>1$. Then, system (4) is globally fixed-time stable, when

$$
t \geq \frac{1}{\beta(q-1)}+\frac{1}{\gamma(1-p)}
$$

Lemma 3. [42] For any vector $x \in R^{n}$ and $0<r<l$

$$
\|x\|_{l} \leq\|x\|_{r} \leq n^{\frac{1}{r}-\frac{1}{l}}\|x\|_{l}
$$

$\left(A_{1}\right)$ : Function $f_{i}: R^{n} \rightarrow R^{n}, i \in \mathbb{L}$, satisfies the following QUAD condition, denoted as $f_{i} \in$ $Q U A D\left(\omega_{i}\right)$, if:

$$
(x-y)^{T}\left(f_{i}(x)-f_{i}(y)\right) \leq \omega_{i}(x-y)^{T}(x-y), \omega_{i}>0 .
$$

$\left(A_{2}\right)$ : Assume that $H_{i j}\left(x_{i}, x_{j}\right)$ satisfies the global Lipschitz condition for any $i, j \in \mathbb{L}$, in other words, there are positive constants $\pi_{i}$ and $v_{i}(i \in \mathbb{L})$, such that

$$
\left\|H_{i j}\left(x_{i}, x_{j}\right)-H_{i j}\left(y_{i}, y_{j}\right)\right\| \leq \pi_{i}\left\|x_{i}-y_{i}\right\|+v_{i}\left\|x_{j}-y_{j}\right\|
$$

for any $\left(x_{i}, x_{j}\right),\left(y_{i}, y_{j}\right) \in R^{m_{i}} \times R^{m_{j}}$. 


\section{Main Results}

In this section, the fixed-time synchronization problem of the drive-response systems with impulsive effects are discussed. The main objective of this section is to design appropriate controllers $u_{i}(t)$ for the drive system (3) so that one can synchronize with the response system (2) within fixed-time.

To achieve the control goal, the following controllers are constructed :

$$
u_{i}(t)=-\gamma_{i} \cdot e_{i}(t)-h_{i} \cdot \operatorname{sign}\left[\phi_{i}\left(x_{i}(t)\right)-\phi_{i}\left(y_{i}(t)\right)\right]^{q}-d_{i} \cdot \operatorname{sign}\left[\phi_{i}\left(x_{i}(t)\right)-\phi_{i}\left(y_{i}(t)\right)\right]^{p}, i \in \mathbb{L},
$$

where $\phi_{i}(z)=\left(\phi_{i 1}\left(z_{1}\right), \phi_{i 2}\left(z_{2}\right), \cdots, \phi_{i m_{i}}\left(z_{m_{i}}\right)\right)^{T}$ for $z \in R^{m_{i}}, \gamma_{i}, h_{i}, d_{i}>0$ are constants called control gains, $q>1,0<p<1$ are constants to be determined. 3 .

To ensure the existence, uniqueness of the solution, the nonlinear function $\phi_{i j}(t)$ satisfy Assumption

$\left(A_{3}\right)$ For any $i \in \mathbb{L}$, there are positive constants $\mu_{i}$ and $\sigma_{i}$ such that $\theta_{i} \leq \frac{\phi_{i j}(x)-\phi_{i j}(y)}{x-y} \leq \sigma_{i}, \forall x \neq y \in$ $R, j=1,2, \ldots, m_{i}$.

We denote some notation in the following of this paper:

$$
\begin{aligned}
& \Omega=\operatorname{diag}\left[\omega_{i}\right]_{N}, \Pi=\operatorname{diag}\left[\pi_{i}\right]_{N}, \Gamma=\operatorname{diag}\left[\gamma_{i}\right]_{N}, \\
& \Upsilon=\operatorname{diag}\left[v_{i}\right]_{N}, \bar{B}=\operatorname{diag}\left[\sum_{j=1}^{N} b_{i j}\right]_{N}, \eta_{i}=\frac{c_{i}}{v_{i}}, \\
& \bar{\Pi}=2 \mu I_{N}, \tilde{v}=\prod_{i=1}^{N} v_{i}, \tilde{m}=\sum_{i=1}^{N} m_{i}, \\
& \check{h}=\min \left\{h_{i}, i \in \mathbb{L}\right\}, \check{d}=\min \left\{d_{i}, i \in \mathbb{L}\right\}, \\
& \check{\theta}=\min \left\{\theta_{i}, i \in \mathbb{L}\right\}, \check{\eta}=\min \left\{\eta_{i}, i \in \mathbb{L}\right\} .
\end{aligned}
$$

\subsection{Strongly Connected Digraph}

Theorem 1. Under Assumptions $\left(A_{1}\right)-\left(A_{3}\right)$, if the weighted matrix $\mathrm{B}$ is irreducible and the following assumptions are fulfilled:

$\left(A_{4}\right) \Omega+\rho(\Pi+\Upsilon) \bar{B}-\Gamma \leq 0$

$\left(A_{5}\right) \tilde{\lambda}=\max _{1 \leq i \leq N}\left(-\lambda_{i}^{k}+1\right)^{2} \leq 1$,

then, under the controller (5), the drive-response system (3) and (2) can achieve synchronization within fixed-time, which is

$$
T=\frac{1}{2 \check{h} \check{\theta}^{q}(\tilde{m} \check{\eta} \tilde{v})^{\frac{1-q}{2}}(q-1)}+\frac{1}{2 \check{d} \check{\theta}^{p}(\check{\eta} \tilde{v})^{\frac{1-p}{2}}(1-p)} .
$$

Proof. Select a Lyapunov function as

$$
V(t)=\sum_{i=1}^{N} \tilde{c}_{i} e_{i}^{T}(t) e_{i}(t)
$$

where $\tilde{c}_{i}$ is the cofactor of the $i$ th diagonal element of $\tilde{L}:=\operatorname{diag}\left[v_{i}\right]_{N} \cdot L$. Obviously, $\tilde{c}_{i}=\tilde{v} \eta_{i}$.

The derivative of $V(t)$ along the trajectories of (4) gives that, for $t \neq t_{k}$,

$$
\begin{aligned}
\dot{V}(t)= & \sum_{i=1}^{N} 2 \tilde{c}_{i} e_{i}^{T}(t)\left(f_{i}\left(x_{i}(t)\right)-f_{i}\left(y_{i}(t)\right)\right)+\sum_{i=1}^{N} 2 \tilde{c}_{i} e_{i}^{T}(t) u_{i}(t) \\
& +\rho \sum_{i=1}^{N} \sum_{j=1}^{N} 2 \tilde{c}_{i} e_{i}^{T}(t) b_{i j}\left(H_{i j}\left(x_{i}, x_{j}\right)-H_{i j}\left(y_{i}, y_{j}\right)\right) .
\end{aligned}
$$


In view of $f_{i}(\cdot) \in Q U A D\left(\omega_{i}\right), i \in \mathbb{L}$ and Assumptions $\left(A_{2}\right)\left(A_{3}\right)$, we can show that

$$
\begin{aligned}
\dot{V}(t) \leq & \sum_{i=1}^{N} 2 \tilde{c}_{i} \omega_{i} e_{i}^{T}(t) e_{i}(t)-\sum_{i=1}^{N} 2 \tilde{c}_{i} \gamma_{i} e_{i}(t)^{T} e_{i}(t)+2 \rho \sum_{i=1}^{N} \sum_{j=1}^{N} \tilde{c}_{i} b_{i j}\left\|e_{i}(t)\right\|\left(\pi_{i}\left\|e_{i}(t)\right\|+v_{i}\left\|e_{j}(t)\right\|\right) \\
& -\sum_{i=1}^{N} 2 \tilde{c}_{i} h_{i}\left|e_{i}(t)\right|^{T}\left|\phi_{i}\left(x_{i}(t)\right)-\phi_{i}\left(y_{i}(t)\right)\right|^{q}-\sum_{i=1}^{N} 2 \tilde{c}_{i} d_{i}\left|e_{i}(t)\right|^{T}\left|\phi_{i}\left(x_{i}(t)\right)-\phi_{i}\left(y_{i}(t)\right)\right|^{p} .
\end{aligned}
$$

Since

$2 \sum_{j=1}^{N} b_{i j}\left\|e_{i}(t)\right\|\left(\pi_{i}\left\|e_{i}(t)\right\|+v_{i}\left\|e_{j}(t)\right\|\right) \leq 2\left(\pi_{i}+v_{i}\right) \sum_{j=1}^{N} b_{i j}\left\|e_{i}(t)\right\|^{2}+v_{i} \sum_{j=1}^{N} b_{i j}\left(\left\|e_{j}(t)\right\|^{2}-\left\|e_{i}(t)\right\|^{2}\right)$

By the Assumption $\left(A_{3}\right)$ and Lemma 3 , we obtain

$$
\begin{aligned}
& \sum_{i=1}^{N} \tilde{c}_{i} h_{i}\left|e_{i}(t)\right|^{T}\left|\phi_{i}\left(x_{i}(t)\right)-\phi_{i}\left(y_{i}(t)\right)\right|^{q} \\
= & \sum_{i=1}^{N} \sum_{j=1}^{m_{i}} \tilde{c}_{i} h_{i}\left|e_{i j}(t)\right|\left|\phi_{i j}\left(x_{i j}(t)\right)-\phi_{i j}\left(y_{i j}(t)\right)\right|^{q} \\
\geq & \sum_{i=1}^{N} \sum_{j=1}^{m_{i}} \tilde{c}_{i} h_{i} \theta_{i}^{q}\left|e_{i j}(t)\right|^{q+1} \\
\geq & \breve{h} \ddot{\theta}^{q} \tilde{m}^{\frac{1-q}{2}}(\check{\eta} \tilde{v})^{\frac{1-q}{2}}\left(\sum_{i=1}^{N} \sum_{j=1}^{m_{i}} \tilde{c}_{i}\left|e_{i j}(t)\right|^{2}\right)^{\frac{q+1}{2}}, \\
& \sum_{i=1}^{N} \tilde{c}_{i} d_{i}\left|e_{i}(t)\right|^{T}\left|\phi_{i}\left(x_{i}(t)\right)-\phi_{i}\left(y_{i}(t)\right)\right|^{p} \\
= & \sum_{i=1}^{N} \sum_{j=1}^{m_{i}} \tilde{c}_{i} d_{i}\left|e_{i j}(t)\right|\left|\phi_{i j}\left(x_{i j}(t)\right)-\phi_{i j}\left(y_{i j}(t)\right)\right|^{p} \\
\geq & \sum_{i=1}^{N} \sum_{j=1}^{m_{i}} \tilde{c}_{i} d_{i} \theta_{i}^{p}\left|e_{i j}(t)\right|^{p+1} \\
\geq & \check{d} \ddot{\theta}^{p}(\check{\eta} \tilde{v})^{\frac{1-p}{2}}\left(\sum_{i=1}^{N} \sum_{j=1}^{m_{i}} \tilde{c}_{i}\left|e_{i j}(t)\right|^{2}\right)^{\frac{p+1}{2}},
\end{aligned}
$$

By the inequalities (7)-(9), yields

$$
\begin{aligned}
\dot{V}(t) \leq & \sum_{i=1}^{N} 2 \tilde{c}_{i} \omega_{i} e_{i}^{T}(t) e_{i}(t)-\sum_{i=1}^{N} 2 \tilde{c}_{i} \gamma_{i} e_{i}(t)^{T} e_{i}(t)+2 \rho \sum_{i=1}^{N} \sum_{j=1}^{N} \tilde{c}_{i}\left(\pi_{i}+v_{i}\right) b_{i j}\left\|e_{i}(t)\right\|^{2} \\
& +\rho \sum_{i=1}^{N} \sum_{j=1}^{N} \tilde{c}_{i} v_{i} b_{i j}\left(\left\|e_{j}(t)\right\|^{2}-\left\|e_{i}(t)\right\|^{2}\right)-2 \check{h} \check{\theta}^{q} \tilde{m}^{\frac{1-q}{2}}(\check{\eta} \tilde{v})^{\frac{1-q}{2}}\left(\sum_{i=1}^{N} \sum_{j=1}^{m_{i}} \tilde{c}_{i}\left|e_{i j}(t)\right|^{2}\right)^{\frac{q+1}{2}} \\
& -2 \check{d} \check{\theta}^{p}(\check{\eta} \tilde{v})^{\frac{1-p}{2}}\left(\sum_{i=1}^{N} \sum_{j=1}^{m_{i}} \tilde{c}_{i}\left|e_{i j}(t)\right|^{2}\right)^{\frac{p+1}{2}} \\
= & 2 \tilde{e}(t)^{T} \tilde{C}(\Omega+\rho(\Pi+\Upsilon) \bar{B}-\Gamma) \tilde{e}(t)+\rho \sum_{i=1}^{N} \sum_{j=1}^{N} \tilde{c}_{i} v_{i} b_{i j}\left(\left\|e_{j}(t)\right\|^{2}-\left\|e_{i}(t)\right\|^{2}\right) \\
& -2 \check{h} \check{\theta}^{q} \tilde{m}^{\frac{1-q}{2}}(\check{\eta} \tilde{v})^{\frac{1-q}{2}} V^{\frac{q+1}{2}}(t)-2 \check{d} \check{\theta}^{p}(\check{\eta} \tilde{v})^{\frac{1-p}{2}} V^{\frac{p+1}{2}}(t) .
\end{aligned}
$$


where $\tilde{e}(t)=\left(\left\|e_{1}(t)\right\|,\left\|e_{2}(t)\right\|, \ldots,\left\|e_{N}(t)\right\|\right)^{T}, \tilde{C}=\operatorname{diag}\left[c_{i}\right]_{N}$.

By the Assumption $\left(A_{4}\right)$, one can obtain

$$
\dot{V}(t) \leq \rho \sum_{i=1}^{N} \sum_{j=1}^{N} \tilde{c}_{i} v_{i} b_{i j}\left(\left\|e_{j}(t)\right\|^{2}-\left\|e_{i}(t)\right\|^{2}\right)-2 \check{h} \check{\theta}^{q} \tilde{m}^{\frac{1-q}{2}}(\check{\eta} \tilde{v})^{\frac{1-q}{2}} V^{\frac{q+1}{2}}(t)-2 \check{d} \check{\theta}^{p}(\check{\eta} \tilde{v})^{\frac{1-p}{2}} V^{\frac{p+1}{2}}(t) .
$$

By the difinition of $\tilde{c}_{i}$ and the lemma 1, yields

$$
\dot{V}(t) \leq-2 \check{h} \check{\theta} \check{\theta}^{q}(\tilde{m} \check{\eta} \tilde{v})^{\frac{1-q}{2}} V^{\frac{q+1}{2}}(t)-2 \check{d} \check{\theta} \check{\theta}^{p}(\check{\eta} \tilde{v})^{\frac{1-p}{2}} V^{\frac{p+1}{2}}(t) .
$$

When $t=t_{k}$, it can be obtain from (6) that

$$
\begin{aligned}
V\left(t_{k}\right) & =\sum_{i=1}^{N} \tilde{c}_{i} e_{i}^{T}\left(t_{k}\right) e_{i}\left(t_{k}\right) \\
& =\sum_{i=1}^{N} \tilde{c}_{i}\left(-\lambda_{i}^{k}+1\right)^{2} e_{i}^{T}\left(t_{k}^{-}\right) e_{i}\left(t_{k}^{-}\right) \\
& \leq \tilde{\lambda} \sum_{i=1}^{N} \tilde{c}_{i} e_{i}^{T}\left(t_{k}^{-}\right) e_{i}\left(t_{k}^{-}\right) \\
& \leq V\left(t_{k}\right)
\end{aligned}
$$

where $\tilde{\lambda}=\max _{1 \leq i \leq N}\left(-\lambda_{i}^{k}+1\right)^{2}$.

According to Lemma 2 , the drive system (1) is globally synchronized with response systems (2) within fixed time, which is

$$
T=\frac{1}{2 \check{h}^{q} \check{\theta}^{q}(\tilde{m} \check{\eta} \tilde{v})^{\frac{1-q}{2}}(q-1)}+\frac{1}{2 \check{d} \check{d}^{p}(\check{\eta} \tilde{v})^{\frac{1-p}{2}}(1-p)} .
$$

The proof is completed.

Especially, when $\pi_{i}=v_{i}=\mu \geq 0$ for $i \in \mathbb{L}$, the Assumption $\left(A_{2}\right)$ is instead by the following assumption:

$\left(A_{2}^{\prime}\right) H_{i j}\left(x_{i}, x_{j}\right)$, for any $i, j \in \mathbb{L}$, satisfies

$$
\left\|H_{i j}\left(x_{i}, x_{j}\right)-H_{i j}\left(y_{i}, y_{j}\right)\right\| \leq \mu\left\|x_{i}-y_{i}\right\|+\mu\left\|x_{j}-y_{j}\right\|,
$$

for any $\left(x_{i}, x_{j}\right),\left(y_{i}, y_{j}\right) \in \mathbb{R}^{m_{i}} \times \mathbb{R}^{m_{j}}$.

Accordingly, we have the following result.

Corollary 1. Assume the weighted matrix B is irreducible. Suppose that Assumptions $\left(A_{1}\right)\left(A_{2}^{\prime}\right)\left(A_{3}\right)$ hold and the following assumptions are fulfilled:

$\left(A_{4}^{\prime}\right) \Omega+\rho \bar{\Pi} \bar{B}-\Gamma \leq 0 ;$

$\left(A_{5}\right) \tilde{\lambda}=\max _{1 \leq i \leq N}\left(-\lambda_{i}^{k}+1\right)^{2} \leq 1$.

Then, under the controller (5), the drive-response systems (3) and (2) can realise synchronisation within a fixed-time

$$
T=\frac{1}{2 \check{h} \check{\theta} q(\tilde{m} \check{\eta} \tilde{v})^{\frac{1-q}{2}}(q-1)}+\frac{1}{2 \check{d} \check{\theta}^{p}(\check{\eta} \tilde{v})^{\frac{1-p}{2}}(1-p)} .
$$

Remark 1. If $H_{i j}\left(x_{i}(t), x_{j}(t)\right)$ and $H_{i j}\left(y_{i}(t), y_{j}(t)\right)$, for any $\left(x_{i}, x_{j}\right),\left(y_{i}, y_{j}\right) \in \mathbb{R}^{m_{i}} \times \mathbb{R}^{m_{j}}$, in driveresponse systems (3) and (2) are replaced by $\Lambda\left(x_{j}-x_{i}\right)$ and $\Lambda\left(y_{j}-y_{i}\right)$, respectively, the converted system will be complex network. Then the result of corollary 1 hold with $\mu=\|\Lambda\|$. 


\subsection{Without Strongly Connected Digraph}

Theorem 2. Let Assumptions $\left(A_{1}\right)-\left(A_{3}\right)$ hold, assume further that

$\left(A_{5}\right) \tilde{\lambda}=\max _{1 \leq i \leq N}\left(-\lambda_{i}^{k}+1\right)^{2} \leq 1$

$\left(A_{6}\right) \Omega+\rho \Pi \bar{B}+\rho \Upsilon B^{s}-\Gamma \leq 0$.

Then, under the controllers (5), the controlled system (3) is said to be synchronized with the response system (2) within a fixed time

$$
T=\frac{1}{2 \check{h}^{q} \tilde{m}^{\frac{1-q}{2}}(q-1)}+\frac{1}{2 \check{d}^{p}(1-p)},
$$

Proof. Select a Lyapunov function as

$$
V(t)=\sum_{i=1}^{N} e_{i}^{T}(t) e_{i}(t) .
$$

The derivative of $V(t)$ along the trajectories of (4) gives that, for $t \neq t_{k}$,

$$
\begin{aligned}
\dot{V}(t)= & \sum_{i=1}^{N} 2 e_{i}^{T}(t)\left(f_{i}\left(x_{i}(t)\right)-f_{i}\left(y_{i}(t)\right)\right)+\sum_{i=1}^{N} 2 e_{i}^{T}(t) u_{i}(t) \\
& +\rho \sum_{i=1}^{N} \sum_{j=1}^{N} 2 e_{i}^{T}(t) b_{i j}\left(H_{i j}\left(x_{i}, x_{j}\right)-H_{i j}\left(y_{i}, y_{j}\right)\right) .
\end{aligned}
$$

In view of $f_{i}(\cdot) \in Q U A D\left(\omega_{i}\right), i \in \mathbb{L}$ and Assumptions $\left(A_{2}\right)\left(A_{3}\right)$, we can show that

$$
\begin{aligned}
\dot{V}(t) \leq & \sum_{i=1}^{N} 2 \omega_{i} e_{i}^{T}(t) e_{i}(t)-\sum_{i=1}^{N} 2 \gamma_{i} e_{i}(t)^{T} e_{i}(t)+2 \rho \sum_{i=1}^{N} \sum_{j=1}^{N} b_{i j}\left\|e_{i}(t)\right\|\left(\pi_{i}\left\|e_{i}(t)\right\|+v_{i}\left\|e_{j}(t)\right\|\right) \\
& -\sum_{i=1}^{N} 2 h_{i}\left|e_{i}(t)\right|^{T}\left|\phi_{i}\left(x_{i}(t)\right)-\phi_{i}\left(y_{i}(t)\right)\right|^{q}-\sum_{i=1}^{N} 2 d_{i}\left|e_{i}(t)\right|^{T}\left|\phi_{i}\left(x_{i}(t)\right)-\phi_{i}\left(y_{i}(t)\right)\right|^{p} .
\end{aligned}
$$

By the Assumption $\left(A_{3}\right)$ and Lemma 3, we obtain

$$
\begin{aligned}
& \sum_{i=1}^{N} h_{i}\left|e_{i}(t)\right|^{T}\left|\phi_{i}\left(x_{i}(t)\right)-\phi_{i}\left(y_{i}(t)\right)\right|^{q} \\
= & \sum_{i=1}^{N} \sum_{j=1}^{m_{i}} h_{i}\left|e_{i j}(t)\right|\left|\phi_{i j}\left(x_{i j}(t)\right)-\phi_{i j}\left(y_{i j}(t)\right)\right|^{q} \\
\geq & \sum_{i=1}^{N} \sum_{j=1}^{m_{i}} h_{i} \theta_{i}^{q}\left|e_{i j}(t)\right|^{q+1} \\
\geq & \breve{h} \check{\theta}^{q} \tilde{m}^{\frac{1-q}{2}}\left(\sum_{i=1}^{N} \sum_{j=1}^{m_{i}}\left|e_{i j}(t)\right|^{2}\right)^{\frac{q+1}{2}}
\end{aligned}
$$




$$
\begin{aligned}
& \sum_{i=1}^{N} d_{i}\left|e_{i}(t)\right|^{T}\left|\phi_{i}\left(x_{i}(t)\right)-\phi_{i}\left(y_{i}(t)\right)\right|^{p} \\
= & \sum_{i=1}^{N} \sum_{j=1}^{m_{i}} d_{i}\left|e_{i j}(t)\right|\left|\phi_{i j}\left(x_{i j}(t)\right)-\phi_{i j}\left(y_{i j}(t)\right)\right|^{p} \\
\geq & \sum_{i=1}^{N} \sum_{j=1}^{m_{i}} d_{i} \theta_{i}^{p}\left|e_{i j}(t)\right|^{p+1} \\
\geq & \breve{d} \breve{\theta}^{p}\left(\sum_{i=1}^{N} \sum_{j=1}^{m_{i}}\left|e_{i j}(t)\right|^{2}\right)^{\frac{p+1}{2}},
\end{aligned}
$$

By the inequalities (11) and (12), yields

$$
\begin{aligned}
\dot{V}(t) \leq & \sum_{i=1}^{N} 2 \omega_{i} e_{i}^{T}(t) e_{i}(t)-\sum_{i=1}^{N} 2 \gamma_{i} e_{i}(t)^{T} e_{i}(t)+2 \rho \sum_{i=1}^{N} \sum_{j=1}^{N} b_{i j}\left\|e_{i}(t)\right\|\left(\pi_{i}\left\|e_{i}(t)\right\|+v_{i}\left\|e_{j}(t)\right\|\right) \\
& -2 \check{h} \check{\theta}^{q} \tilde{m}^{\frac{1-q}{2}}\left(\sum_{i=1}^{N} \sum_{j=1}^{m_{i}}\left|e_{i j}(t)\right|^{2}\right)^{\frac{q+1}{2}}-2 \check{d} \check{\theta}^{p}\left(\sum_{i=1}^{N} \sum_{j=1}^{m_{i}}\left|e_{i j}(t)\right|^{2}\right)^{\frac{p+1}{2}} \\
= & 2 \tilde{e}(t)^{T}\left(\Omega+\rho \Pi \bar{B}+\rho \Upsilon B^{s}-\Gamma\right) \tilde{e}(t)-2 \check{h} \check{\theta}^{q} \tilde{m}^{\frac{1-q}{2}} V^{\frac{q+1}{2}}(t)-2 \breve{d} \breve{\theta}^{p} V^{\frac{p+1}{2}}(t) .
\end{aligned}
$$

where $\tilde{e}(t)=\left(\left\|e_{1}(t)\right\|,\left\|e_{2}(t)\right\|, \ldots,\left\|e_{N}(t)\right\|\right)^{T}$.

When $t=t_{k}$, it can be obtain from (10) that

$$
\begin{aligned}
V\left(t_{k}\right) & =\sum_{i=1}^{N} e_{i}^{T}\left(t_{k}\right) e_{i}\left(t_{k}\right) \\
& =\sum_{i=1}^{N}\left(-\lambda_{i}^{k}+1\right)^{2} e_{i}^{T}\left(t_{k}^{-}\right) e_{i}\left(t_{k}^{-}\right) \\
& \leq \tilde{\lambda} \sum_{i=1}^{N} e_{i}^{T}\left(t_{k}^{-}\right) e_{i}\left(t_{k}^{-}\right) \\
& \leq V\left(t_{k}\right)
\end{aligned}
$$

where $\tilde{\lambda}=\max _{1 \leq i \leq N}\left(-\lambda_{i}^{k}+1\right)^{2}$.

According to Lemma 2 , the drive system (3) is globally synchronized with response systems (2) within fixed time, which is

$$
T=\frac{1}{2 \check{h} \check{\theta}^{q} \tilde{m}^{\frac{1-q}{2}}(q-1)}+\frac{1}{2 \check{d} \check{\theta}^{p}(1-p)} .
$$

The proof is completed.

Corollary 2. let the Assumptions $\left(A_{1}\right)-\left(A_{3}\right)$ hold, except that Assumption $\left(A_{2}\right)$ is replaced by the $\left(A_{2}^{\prime}\right)$. Assume further that

$$
\begin{aligned}
& \left(A_{5}\right) \tilde{\lambda}=\max _{1 \leq i \leq N}\left(-\lambda_{i}^{k}+1\right)^{2} \leq 1 ; \\
& \left(A_{6}^{\prime}\right) \Omega+\frac{1}{2} \rho \bar{\Pi} \overline{\bar{B}}+\frac{1}{2} \rho \bar{\Pi} B^{s}-\Gamma \leq 0 .
\end{aligned}
$$

Then, under the controllers (5), the drive-response systems (3) and (2) are synchronized within a fixed time

$$
T=\frac{1}{2 \check{h} \check{\theta}^{q} \tilde{m}^{\frac{1-q}{2}}(q-1)}+\frac{1}{2 \check{d} \check{\theta}^{p}(1-p)} .
$$


Remark 2. Especially, considering the complex network, which means $H_{i j}\left(x_{i}, x_{j}\right)=\Lambda\left(x_{j}-x_{i}\right), H_{i j}\left(y_{i}(t)\right.$, $\left.y_{j}(t)\right)=\Lambda\left(y_{j}-y_{i}\right)$, for any $\left(x_{i}, x_{j}\right),\left(y_{i}, y_{j}\right) \in \mathbb{R}^{m_{i}} \times \mathbb{R}^{m_{j}}, i, j \in \mathbb{L}$ in drive-response systems (3) and (2). Then the corollary 2 hold with $\mu=\|\Lambda\|$. For this reason, the model in [31] is special cases of this work. And Corollary 2 extends those corresponding theoretical results in [31].

Remark 3. Note that we do not assume that the weight configuration matrix B is irreducible or symmetric in Theorem 2. So the result of Theorem 2 can be used in many models. Moreover, the inner coupling matrix $\Lambda$, which is identity matrix in [33], is not assumed to be diagonal or positive definite in our paper. Therefore, the corollary 2 is more general than these in [33].

Remark 4. In the literature, finite time synchronization, whose convergence time is attached to the original states of system, was studied in many papers, see [26]. In this paper, the fixed-time controllers are introduced to synchronize DRCS with impulsive effects, which means that better performance of the system can be achieved. From the perspective of practicality, the fixed-time controllers are more widely used.

Remark 5. Suppose $\phi_{i}(x)=x$. Then the controllers Eq.(5) can be

$$
u_{i}(t)=-\gamma_{i} \cdot e_{i}(t)-h_{i} \cdot \operatorname{sign}\left[e_{i}(t)\right]^{q}-d_{i} \cdot \operatorname{sign}\left[e_{i}(t)\right]^{p},
$$

which is consistent with the controllers in [37]. For this reason, the controllers in this paper are less conservative than ones in [37]. What's more, [37] didn't consider the topological structure of $(\mathcal{G}, B)$. Therefore, our results are more rich than its.

\section{Numerical Example}

In this section, some simulation examples are given to verify the effectiveness and feasibility of the criteria established above.

For this purpose, we study the complex network and consider Rössler-like system [43] as the node dynamics.

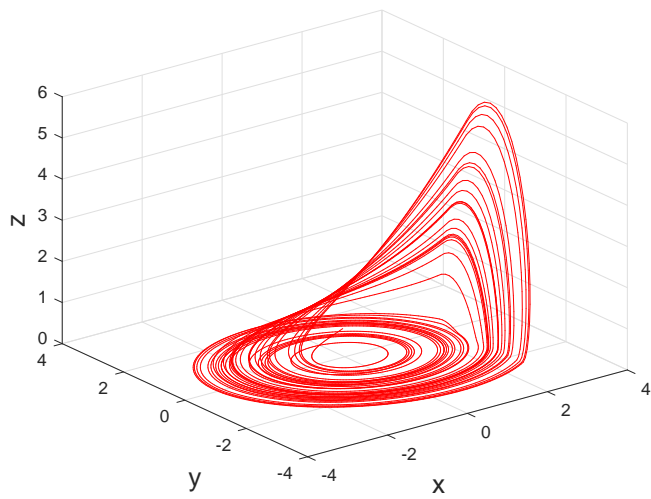

Figure 1. The chaotic trajectories of the Rössler-like system with initial values $(0.3,0.4,0.5)$.

The Rössler-like system is presented as

$$
\dot{x}=f(x)=\alpha\left(\begin{array}{ccc}
-\Gamma & -\beta & -\lambda \\
1 & \gamma & 0 \\
0 & 0 & -\mu
\end{array}\right)\left(\begin{array}{l}
x_{1} \\
x_{2} \\
x_{3}
\end{array}\right)+\left(\begin{array}{c}
0 \\
0 \\
\alpha \mu \psi\left(x_{1}\right)
\end{array}\right),
$$

where $x=\left(x_{1}, x_{2}, x_{3}\right)^{T} \in R^{3}$ is the state vector 


$$
\psi(s)= \begin{cases}0, & s<2.56 \\ \xi(s-2.56), & s \geq 2.56\end{cases}
$$

which has a chaotic attractor when $\alpha=0.03, \Gamma=0.075, \beta=1.5, \lambda=0.75, \gamma=0.2, \mu=1.5$, and $\xi=21.43$, see Fig.1.

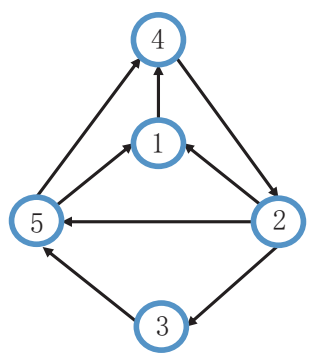

Figure 2. A simple directed complex network with five nodes.

In this section, the following derive-response complex dynamical network with impulsive effects is considered:

$$
\left\{\begin{array}{l}
\dot{x}_{i}(t)=f_{i}\left(x_{i}(t)\right)+\rho \sum_{j=1}^{5} b_{i j} \Lambda x_{j}(t)+u_{i}(t), t \neq t_{k} \\
\triangle x_{i}\left(t_{k}\right)=-d_{i}^{k} e_{i}\left(t_{k}^{-}\right), t=t_{k} \\
\dot{y}_{i}(t)=f_{i}\left(y_{i}(t)\right)+\rho \sum_{j=1}^{5} b_{i j} \Lambda y_{j}(t) .
\end{array}\right.
$$

where

$$
f_{i}\left(x_{i}(t)\right)=f(x), f_{i}\left(y_{i}(t)\right)=f(x), i=1,2, \ldots, 5,
$$

the coupling strength $\rho=1, B$ is an irreducible matrix(see Fig.2).

$$
B=\left(\begin{array}{lllll}
0 & 1 & 0 & 0 & 1 \\
0 & 0 & 0 & 1 & 0 \\
0 & 1 & 0 & 0 & 0 \\
1 & 0 & 0 & 0 & 1 \\
0 & 1 & 1 & 0 & 0
\end{array}\right), \Lambda=\left(\begin{array}{lll}
2 & 1 & 0 \\
0 & 2 & 1 \\
1 & 1 & 0
\end{array}\right)
$$

the controllers $\phi_{i}(x)=x$ for $i=1,2, \ldots, 5$ and $x \in R^{3}$. Therefore, $\mu=\|\Lambda\|=2.9746, \bar{\Pi}=5.9492 I_{5}$, $\theta_{i}=1, \bar{B}=\operatorname{diag}[2,1,1,2,2], \lambda_{i}^{k}=0.5$. And there exist $\omega_{i}=0.492$, for $i=1,2, \ldots, 5$, satisfying $\left(A_{1}\right)$ for the analysis of [43]. Hence, $\Delta=0.492 I_{5}$. If $\gamma_{i} \geq 7, i=1,2, \ldots, 5$, the left side of $\left(A_{4}^{\prime}\right)$ is a negative definite matrix. Hence, the system (13) can achieve synchronization within the estimated fixed-time according to Corollary 1.

Under the controllers Eq.(5) with $\gamma_{i}=7, h_{i}=5, d_{i}=5, q=1.5, p=0.3$, the system (13) can achieve synchronization within $T=0.0531$ by Corollary 1 (see Fig. 3 ). 


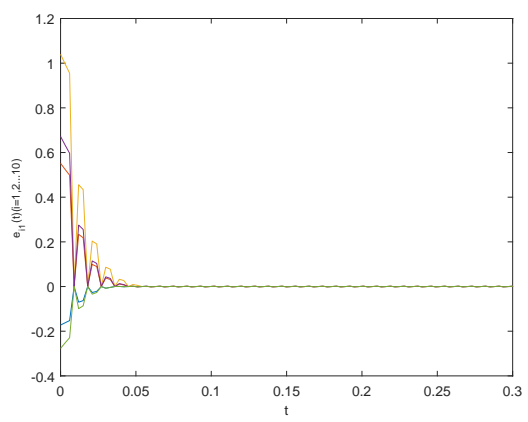

(a)
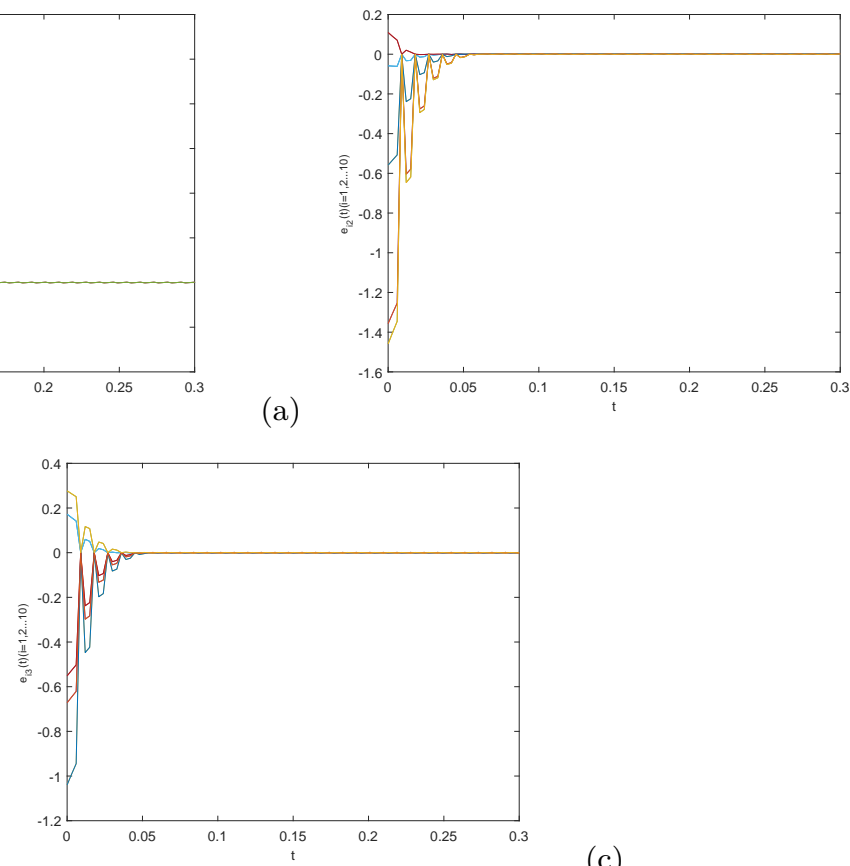

(b)

(c)

Figure 3. The trajectories of the complex system with $x_{i}(0)=\left[e^{-i}, 0.1 i, \cos i\right], y_{i}(0)=\left[\cos i, 1-\sin i, e^{-i}\right]$.

Remark 6. In [44], synchronization method for Rössler chaotic system was constructed and applied to secure transmission of speech and images under the impulsive control, but the time of synchronization is not sure. our result can handle this problem shown in numerical example. Therefore, our result is better applied in communication than the result in [44].

\section{Conclusions}

In this paper, considering the effects of impulsive effects, fixed-time synchronization of derive-response coupled system was studied. By using Lyapunov stability theory and designing more general controllers, sufficient conditions were obtained to ensure synchronization of DRCS with impulsive effects within fixed-time. Finally, some numerical simulations were presented. It is worth noting that the digraphs with strongly connected and without strongly connected were studied in section 2 . A future work is to study the dynamical systems with the harmful impulses to extend the theorems established in the paper. Besides, time delays are unavoidable for real DRCS. Hence, the fixed-time synchronization of impulsive dynamical networks with time delayed will be challenging.

\section{References}

1. M. Y. Li and Z. Shuai, "Global-stability problem for coupled systems of differential equations on networks," J. Differential Equations, vol. 248, no. 1, pp. 1-20, JAN 12010.

2. D. Ganji and A. Sadighi, "Application of He's homotopy-perturbation method to nonlinear coupled systems of reaction-diffusion equations," Int. J. Nonlinear Sci. Numer, vol. 7, no. 4, pp. 411-418, 2006.

3. M. Liu and C. Bai, "Optimal harvesting of a stochastic delay competitive model," Discrete Contin. Dyn. Syst. Ser. B, vol. 22, no. 4, pp. 1493-1508, JUN 2017.

4. E. G. Lakatta, V. A. Maltsev, and T. M. Vinogradova, "A Coupled SYSTEM of Intracellular Ca2+ Clocks and Surface Membrane Voltage Clocks Controls the Timekeeping Mechanism of the Heart's Pacemaker," Circ. Res., vol. 106, no. 4, pp. 659-673, MAR 52010. 
5. M. Liu and C. Bai, "Analysis of a stochastic tri-trophic food-chain model with harvesting," J. Math. Biol., vol. 73, no. 3, pp. 597-625, SEP 2016.

6. J. Shi, P. Zhang, D. Xiao, and Q. Niu, "Proper definition of spin current in spin-orbit coupled systems," Phys. Rev. Lett., vol. 96, no. 7, FEB 242006.

7. Z. N. Low, R. A. Chinga, R. Tseng, and J. Lin, "Design and Test of a High-Power High-Efficiency Loosely Coupled Planar Wireless Power Transfer System," IEEE Trans. Ind. Electron., vol. 56, no. 5, pp. 1801-1812, MAY 2009.

8. D. Ye, X. Yang, and L. Su, "Fault-tolerant synchronization control for complex dynamical networks with semi-Markov jump topology," Appl. Math. Comput., vol. 312, pp. 36-48, NOV 12017.

9. R. Pastor-Satorras, C. Castellano, P. Van Mieghem, and A. Vespignani, "Epidemic processes in complex networks," Rev. Mod. Phys., vol. 87, no. 3, pp. 925-979, AUG 312015.

10. R. Guo, Z. Zhang, X. Liu, and C. Lin, "Existence, uniqueness, and exponential stability analysis for complexvalued memristor-based BAM neural networks with time delays," Appl. Math. Comput., vol. 311, pp. 100-117, OCT 152017.

11. S. Boccaletti, J. Kurths, G. Osipov, D. Valladares, and C. Zhou, "The synchronization of chaotic systems," Phys. Rep., vol. 366, no. 1, pp. 1-101, 2002.

12. L. Wang, Z. Wang, Q.-L. Han, and G. Wei, "Synchronization Control for a Class of Discrete-Time Dynamical Networks With Packet Dropouts: A Coding-Decoding-Based Approach," IEEE Trans. Cybern., vol. 48, no. 8, pp. 2437-2448, AUG 2018.

13. Z. Wu and H. Leng, "Complex hybrid projective synchronization of complex-variable dynamical networks via open-plus-closed-loop control," J. Franklin Inst, vol. 354, no. 2, pp. 689-701, JAN 2017.

14. M. Liu, H. Jiang, and C. Hu, "New Results for Exponential Synchronization of Memristive Cohen-Grossberg Neural Networks with Time-Varying Delays," Neural Processing Letters, vol. 49, no. 1, pp. 79-102, FEB 2019.

15. Y. Wu, B. Chen, and W. Li, "Synchronization of stochastic coupled systems via feedback control based on discrete-time state observations," Nonlinear Anal-Hybri, vol. 26, pp. 68-85, NOV 2017.

16. W. Wu and T. Chen, "Global synchronization criteria of linearly coupled neural network systems with timevarying coupling," IEEE Trans. Neural Netw., vol. 19, no. 2, pp. 319-332, FEB 2008.

17. A. Chandrasekar, R. Rakkiyappan, J. Cao, and S. Lakshmanan, "Synchronization of memristor-based recurrent neural networks with two delay components based on second-order reciprocally convex approach," Neural Netw, vol. 57, pp. 79-93, SEP 2014.

18. B. Guo, Y. Wu, Y. Xiao, and C. Zhang, "Graph-theoretic approach to synchronizing stochastic coupled systems with time-varying delays on networks via periodically intermittent control," Appl. Math. Comput., vol. 331, pp. 341-357, AUG 152018.

19. A. Chandrasekar, R. Rakkiyappan, and J. Cao, "Impulsive synchronization of Markovian jumping randomly coupled neural networks with partly unknown transition probabilities via multiple integral approach," Neural Netw, vol. 70, pp. 27-38, OCT 2015.

20. C. Li, W. Sun, and J. Kurths, "Synchronization between two coupled complex networks," PHYSICAL REVIEW E, 2007.

21. L.M.Pecora and T.L.carroll, "Synchronization chaotic system," Phys.Lett, vol. 64, no. 64, pp. 821-824, 1990.

22. Y. Liu, C. Zhu, D. Chu, and W. Li, "Synchronization of stochastic coupled systems with time-varying coupling structure on networks via discrete-time state feedback control," Neurocomputing, vol. 285, pp. 104-116, APR 122018.

23. V. Haimo, "Finite-time controllers," SIAM J. Control Optim, vol. 24, no. 4, pp. 760-770, 1986.

24. S. Bowong and F. Kakmeni, "Chaos control and duration time of a class of uncertain chaotic systems," Phys. Lett. A, vol. 316, no. 3-4, pp. 206-217, SEP 222003.

25. Z.-H. Guan, F.-L. Sun, Y.-W. Wang, and T. Li, "Finite-Time Consensus for Leader-Following Second-Order Multi-Agent Networks," IEEE Trans. Circuits Syst. I, vol. 59, no. 11, pp. 2646-2654, NOV 2012.

26. J. Yang, Xinsong; Lu, "Finite-time synchronization of coupled networks with markovian topology and impulsive effects," IEEE Transactions On Automatic Control, vol. 61, no. 8, pp. 2256-2261, Aug. 2016.

27. W. Zhang, X. Yang, C. Xu, J. Feng, and C. Li, "Finite-Time Synchronization of Discontinuous Neural Networks With Delays and Mismatched Parameters," IEEE Trans. Neural Netw. Learn. Syst., vol. 29, no. 8, pp. 3761-3771, AUG 2018.

28. J. Li, G. Wei, D. Ding, and Y. Li, "Finite-time control in probability for time-varying systems with measurement censoring," J. Franklin Inst, vol. 356, no. 4, pp. 1677-1694, MAR 2019.

29. A. Polyakov, "Nonlinear Feedback Design for Fixed-Time Stabilization of Linear Control Systems," IEEE Trans. Autom. Control, vol. 57, no. 8, SI, pp. 2106-U1, AUG 2012.

30. W. Zhang, C. Li, T. Huang, and J. Huang, "Fixed-time synchronization of complex networks with nonidentical nodes and stochastic noise perturbations," Physica A, vol. 492, pp. 1531-1542, FEB 152018. 
31. J. H. D. W. C. Yang, Xinsong; Lam, "Fixed-time synchronization of complex networks with impulsive effects via nonchattering control," IEEE Transactions On Automatic Control, vol. 62, no. 11, pp. 5511-5521, Nov. 2017.

32. Y. Wan, J. Cao, G. Wen, and W. Yu, "Robust fixed-time synchronization of delayed cohen-grossberg neural networks," Neural Networks, vol. 73, pp. 86-94, JAN 2016.

33. J. Liu, Y. Yu, Q. Wang, and C. Sun, "Fixed-time event-triggered consensus control for multi-agent systems with nonlinear uncertainties," Neurocomputing, vol. 260, pp. 497-504, OCT 182017.

34. J. C. J. Lu, D. W. C. Ho, "Synchronization of delayed complex dynamical networks with impulsive and stochastic effects," Automatica, vol. 46, pp. 1215-1221, 2010.

35. X. S. A. Khadra, X. Liu, "Application of impulsive synchronization to communication security vol." IEEE Trans. Circuits Syst. I, vol. 50, no. 3, pp. 341-351, 2003.

36. C. W. X. Li, M. Bohner, "Impulsive differential equations: Periodic solutions and applications," Automatica, vol. 52 , pp. $173-178,2015$.

37. T. W. H.Li, C.Li, "Fixed-time stabilization of impulsive cohen-grossberg bam neural networks," Neural Netw, vol. 98, pp. 203-211, 2018.

38. Y. W. X. Zhang, Wenbing; Tang, "Synchronization of nonlinear dynamical networks with heterogeneous impulses," IEEE Transactions on circuits and systems1-regular papers, vol. 61, no. 4, pp. 1220-1228, Apr. 2014.

39. F. L. J. He, Wangli; Qian, "Quasi-synchronization of heterogeneous dynamic networks via distributed impulsive control: Error estimation, optimization and design," Automatica, vol. 63, pp. 249-262, Dec. 2015.

40. J. Li, Xiaodi; Wu, "Stability of nonlinear differential systems with state-dependent delayed impulses," Automatica, vol. 64, pp. 63-69, Feb. 2016.

41. X. Liu, T. Chen, and W. Lu, "Consensus problem in directed networks of multi-agents via nonlinear protocols," Phys. Lett. A, vol. 373, no. 35, pp. 3122-3127, AUG 242009.

42. M.Goldberg, "Equivalence constants constants for i norms of matrices," Linear Multilinear Algebra, vol. 21, no. 2, pp. 173-179, 1987.

43. Y. Sun, W. Li, and D. Zhao, "Finite-time stochastic outer synchronization between two complex dynamical networks with different topologies," Chaos, vol. 22, no. 2, JUN 2012.

44. Q. Yi and Z. H. Shenming Hua, "Impulsive synchronization of rossller chaotic system with applications to communications," JOURNAL OF AIR FORCE ENGINEERING UNIVERSITY, vol. 2, no. 6, pp. 63-65, 2001. 